\title{
Superfluid density near the critical temperature in the presence of random planar defects
}

\author{
D. Dalidovich, A.J. Berlinsky and C. Kallin \\ Department of Physics and Astronomy, McMaster University, \\ Hamilton, Ontario, Canada L8S $4 M 1$
}

(Dated: November 13, 2018)

\begin{abstract}
The superfluid density near the superconducting transition is investigated in the presence of spatial inhomogeneity in the critical temperature. Disorder is accounted for by means of a random $T_{c}$ term in the conventional Ginzburg-Landau action for the superconducting order parameter. Focusing on the case where a low-density of randomly distributed planar defects are responsible for the variation of $T_{c}$, we derive the lowest order correction to the superfluid density in powers of the defect concentration. The correction is calculated assuming a broad Gaussian distribution for the strengths of the defect potentials. Our results are in a qualitative agreement with the superfluid density measurements in the underdoped regime of high-quality YBCO crystals by Broun and coworkers.

PACS numbers:
\end{abstract}

\section{INTRODUCTION}

The superconducting transition measured in real materials is often smeared or broadened in temperature in a way that correlates with sample quality or disorder. A sharper transition is taken as a signature of a higher quality sample. On the other hand, a straightforward application of the Harris criterion $\underline{1}$ implies that uncorrelated disorder is irrelevant and does not affect the nature of the superconducting transition. The Harris criterion is modified for correlated disorder and implies that the transition can be broadened, depending on the nature or dimension of the correlation.

In addition, to the situations considered by Harris, the existence of rare regions (analogous to Lifshitz tails ${ }^{2}$ in the density of states of a disordered semiconductor) with higher than average critical temperatures may also significantly affect the properties close to $T_{c}$ leading to a smeared behavior of the order parameter as the transition is approached from the ordered phases. Such rare regions can occur with or without correlations of the disorder. This type of behavior is expected to be especially pronounced for cases in which the bare superconducting correlation length $\xi_{0}$ is short. High temperature superconductors in the underdoped regime, having a short coherence length at low temperatures, present a good example of systems with increased sensitivity to various types of correlated disorder. Furthermore, many of these materials exhibit twin boundaries, grain boundaries and/or disorder due to oxygen chains, all of which are examples of correlated disorder. Therefore, the high temperature superconductors present a good example of systems with increased sensitivity to various types of disorder.

High- $T_{c}$ superconductors, because they are unconventional in the sense that the gap averages to zero over the Fermi surface, are very sensitive to disorder, although the sensitivity to uncorrelated random disorder is somewhat mitigated by their short coherence lengths. $\stackrel{\underline{3}}{=}$ The role of disorder in superconductors has been an important subject of study for several decades $3 \cdot \underline{3.5}$. Imperfections in the lattice structure are not only responsible for the diffusive motion of charge carriers above $T_{c}$, but also lead to non-uniformity of the attractive interaction between them, ultimately giving rise to a spatial variation of the local critical temperature ${ }^{6.7}$. In addition to structural irregularities, inhomogeneities in $T_{c}$ may be caused by the coexistence of superconductivity and various density waves $\frac{8,9,10}{}$. Indeed, the simultaneous treatment of several competing orders may be at the heart of a complete description of high-temperature superconductors 11,12 and other strongly correlated systems.

Thus, obtaining a comprehensive understanding of all aspects of the complicated interplay between superconductivity and various types of inhomogeneities, is definitely an important goal. An experimentally relevant set of issues that one can specifically address in this context, would include the influence of inhomogeneities on the critical temperature, fluctuation corrections above the transition, as well as the behavior of the order parameter just below $T_{C}$. It is clear that if the characteristic length scale of the inhomogeneity is large compared to the $T=0$ superconducting correlation length, and the width of the distribution of critical temperatures is small compared to some average $T_{C}$, the problem can be studied within the framework of Ginzburg-Landau (GL) theory with a space-dependent critical temperature $T_{c}(\mathbf{r})$. The GL action is suitable for describing universal properties of a system in the vicinity of the critical point which are insensitive to the details of the microscopic Hamiltonian. However, the functional form of $T_{c}(\mathbf{r})$ is non-universal and is determined by the type of non-uniformity present in the system.

In this paper, we study the behavior of the superfluid density near the transition in the presence of one specific type of randomness in $T_{c}$, caused by the presence 
of widely scattered planar defects. The reason for this choice is two-fold. First, twinning planes, that may be present even in high quality crystals, can be regarded as planar defects locally increasing the critical temperature. If a twinning plane is located at $x=0$, the Ginzburg-Landau (GL) action will contain a $\delta$-function term $-u \delta(x)|\Psi(\mathbf{r})|^{2}$ with $u>0 \stackrel{13}{\underline{n}}$ Second, to study the effect of disorder in general, one needs to find stable solutions of the GL equation for arbitrary $T_{c}(\mathbf{r})$ and then average over realizations of disorder in expressions for the physical quantities of interest. This is difficult to accomplish because the GL equation is non-linear. The problem becomes more tractable, however, if the correction to the GL action responsible for the change in $T_{c}$ has the form mentioned above, with $u \gtrless 0$.

In this work, we consider a special case in which the spatial variation of the critical temperature is modeled by randomly located planar defects at points $X_{i}$, each contributing a term

$$
u(\mathbf{r})|\Psi(\mathbf{r})|^{2}=-u \delta\left(x-X_{i}\right)|\Psi(\mathbf{r})|^{2}
$$

to the GL action. Assuming that these imperfections are dilute, we obtain a correction to the uniform superfluid density that is proportional to the defect concentration $n_{i}$. The calculations are performed at the simplest Gaus- sian level of GL theory. The disorder potential $u$ is taken to have a Gaussian distribution with zero average value. The presence of defects that locally enhance the critical temperature, gives rise to a spatially decaying solution for the order parameter around them. The leads to a small but non-zero total transverse response at temperatures greater than $T_{c}^{(0)}$, the critical temperature of the pure superconductor. The actual critical temperature corresponds to the region of strongest $T_{c}$-enhancement and enters as a non-universal parameter in our treatment.

Our work is motivated by recent experiments in the underdoped regime of high-quality YBCO crystals $\underline{14}$ Experiments, done using the cavity perturbation method, show that, despite the high quality of the samples, vestiges of finite superfluid density persist even for temperatures above $T_{c}^{(0)}$. Our findings, based on the assumption of dilute planar inhomogeneities in the form of twin boundaries, qualitatively explain the results of measurements close to the critical temperature.

\section{METHOD OF EXPANSION IN POWERS OF THE DEFECT CONCENTRATION}

The starting point of our calculations close to criticality is the Ginzburg-Landau (GL) free energy in terms of the local superconducting order parameter $\Psi(\mathbf{r})::^{15}$

$$
\mathcal{F}=\mathcal{F}_{n}+\int d \mathbf{r}\left\{\frac{1}{2 m}|\nabla \Psi(\mathbf{r})|^{2}+\alpha(T)|\Psi(\mathbf{r})|^{2}+U(\mathbf{r})|\Psi(\mathbf{r})|^{2}+\frac{b}{2}|\Psi(\mathbf{r})|^{4}\right\}
$$

Here $\mathcal{F}_{n}$ is the free energy of the normal system and

$$
\alpha(T)=a\left(\frac{T-T_{c}^{(0)}}{T_{c}^{(0)}}\right)
$$

is the distance from the critical temperature, $T_{c}^{(0)}$, of a homogeneous superconductor. We assume that the deviations of $T_{c}(\mathbf{r})$ from $T_{c}^{(0)}$, described by $U(\mathbf{r})$, occur in regions of a size greater than or of order the $T=0$ correlation length, $\xi_{0}=\xi(T=0)$, but small compared to the correlation length near $T_{c}^{(0)}$. This assumption justifies the use of the GL formalism for both conventional and short coherence length superconductors, provided that they are not too far from the critical temperature. In this case, if the regions where the critical temperature differs sufficiently from $T_{c}^{(0)}$ are located around points $\mathbf{R}_{i}$, we can quite generally model the randomness in Eq. (2) by

$$
U(\mathbf{r})=\sum_{i} u_{i}\left(\mathbf{r}-\mathbf{R}_{i}\right)
$$

In the subsequent treatment, we will refer to these regions as defects. The functions $u_{i}\left(\mathbf{r}-\mathbf{R}_{i}\right)$, that we will henceforth call the potentials, are presumed to be quickly decaying with $\left|\mathbf{r}-\mathbf{R}_{i}\right|$. From the above considerations, the characteristic lengths of this decay must exceed $\xi_{0}$ but be small compared to the correlation length $\xi(T)$ close enough to the critical temperature. In addition, it must be much smaller than the average separation between the positions of the defects $n_{i}^{-1 / d}$. In the Gaussian approximation of GL theory $\xi(T)=1 / \sqrt{2 m \alpha(T)}$.

The equilibrium distribution of the superconducting order parameter, $\Psi_{0}(\mathbf{r})$, follows from the solution of the saddle point GL equation that is derived by varying Eq. (2) with respect to $\Psi^{*}(\mathbf{r})$ :

$$
\left[-\frac{\nabla^{2}}{2 m}+\alpha(T)+U(\mathbf{r})\right] \Psi_{0}(\mathbf{r})+b\left|\Psi_{0}(\mathbf{r})\right|^{2} \Psi_{0}(\mathbf{r})=0 .(5
$$

For a given disorder potential, the actual transition temperature, $T_{c}$, is determined from the value of $\alpha\left(T_{c}\right)$ for which a non-zero solution of $\Psi_{0}(\mathbf{r})$ first appears. This happens when the eigenvalue spectrum of the operator

$$
\hat{L}_{0}[U]=-\frac{\nabla^{2}}{2 m}+\alpha(T)+U(\mathbf{r})
$$


crosses zero. The ensuing distribution $\Psi_{0}(\mathbf{r})$ can be chosen real and positive everywhere in space and must be stable. The stability conditions can be determined if one expands the generally complex order parameter $\Psi(\mathbf{r})$ around $\Psi_{0}(\mathbf{r})$ in Eq. (2),

$$
\Psi(\mathbf{r})=\Psi_{0}(\mathbf{r})+\Psi_{\|}(\mathbf{r})+i \Psi_{\perp}(\mathbf{r}) .
$$

One can then easily infer that a non-negative eigenspectrum for the operators

$$
\begin{aligned}
& \hat{L}_{\perp}[U]=-\frac{\nabla^{2}}{2 m}+\alpha(T)+U(\mathbf{r})+b \Psi_{0}^{2}(\mathbf{r}), \\
& \hat{L}_{\|}[U]=-\frac{\nabla^{2}}{2 m}+\alpha(T)+U(\mathbf{r})+3 b \Psi_{0}^{2}(\mathbf{r}),
\end{aligned}
$$

is a necessary condition for stability. The problem of determining the transition point and finding the stable solution below the transition analytically for the general form, $U(\mathbf{r})$, is a daunting task. However, one can simplify the problem if the concentration of defects $n_{i}$ is small. If one assumes that every defect potential, $u_{i}\left(\mathbf{r}-\mathbf{R}_{i}\right)$, is characterized by the same set of parameters $\{u\}$, one can employ the method of expansion in powers of concentration $n_{i}, 16$

Consider the function

$$
F_{N}\left(\left\{u_{1}\right\} \mathbf{R}_{1}, \ldots,\left\{u_{N}\right\} \mathbf{R}_{N} ; \mathbf{r}\right)
$$

that describes the spatial dependence of some quantity of interest and is calculable based on the GL action in the presence of $N$ defects. We presume also that every defect located at point $\mathbf{R}_{i}$ has a potential characterized by the specific parameter set $\left\{u_{i}\right\}$. The values of the parameters $\{u\}$ are distributed according to the distribution $\mathcal{P}(\{u\})$, satisfying the normalization condition

$$
\int \mathcal{P}(\{u\}) \mathcal{D}\{u\}=1
$$

In Eq. (9), $\mathcal{D}\{u\}$ formally means the integration over all variables in the set $\{u\}$. We are interested in the value of the function $F_{N}\left(\left\{u_{1}\right\} \mathbf{R}_{1}, \ldots,\left\{u_{N}\right\} \mathbf{R}_{N} ; \mathbf{r}\right)$ that is an average over positions $\mathbf{R}_{i}$ as well as parameters $\left\{u_{i}\right\}$. If one denotes the positional average by angular brackets, the full average can be written in the form:

$$
\overline{F_{N}\left(\left\{u_{1}\right\} \mathbf{R}_{1} \ldots\left\{u_{N}\right\} \mathbf{R}_{N} ; \mathbf{r}\right)}=\int \mathcal{P}\left(\left\{u_{1}\right\}\right) \ldots \mathcal{P}\left(\left\{u_{N}\right\}\right) \mathcal{D}\left\{u_{1}\right\} \ldots \mathcal{D}\left\{u_{N}\right\}\left\langle F_{N}\left(\left\{u_{1}\right\} \mathbf{R}_{1}, \ldots,\left\{u_{N}\right\} \mathbf{R}_{N} ; \mathbf{r}\right)\right\rangle
$$

Regarding the concentration of defects as small, one can formally write the average Eq. (10) in the form of a series in powers of $n_{i}$. The details of the corresponding derivation are presented in Appendix A. As follows from Eqs. (A2)(A4), up to the first order in $n_{i}$,

$$
\left\langle F_{N}\left(\left\{u_{1}\right\} \mathbf{R}_{1}, \ldots,\left\{u_{N}\right\} \mathbf{R}_{N} ; \mathbf{r}\right)\right\rangle=F_{0}(\mathbf{r})+n_{i} \int\left[F_{1}\left(\left\{u_{1}\right\} ; \mathbf{R}_{1} ; \mathbf{r}\right)-F_{0}(\mathbf{r})\right] d \mathbf{R}_{1},
$$

so that

$$
\overline{F_{N}\left(\left\{u_{1}\right\} \mathbf{R}_{1} \ldots\left\{u_{N}\right\} \mathbf{R}_{N} ; \mathbf{r}\right)}=F_{0}(\mathbf{r})+n_{i} \int \mathcal{P}(\{u\}) \mathcal{D}\{u\} \int\left[F_{1}(\{u\} ; \mathbf{R} ; \mathbf{r})-F_{0}(\mathbf{r})\right] d \mathbf{R}
$$

Eq. (12) contains the lowest order correction to the function $F_{0}(\mathbf{r})$, the quantity of interest in the absence of any defects. The calculation of this correction requires the knowledge of function $F_{1}(\{u\} ; \mathbf{R} ; \mathbf{r})-$ the quantity of interest in the presence of just one defect located at point $\mathbf{R}$. We should mention that the approach described in Appendix A, provides a way to reduce the level of complexity of the initial problem, since it reduces to calculations in the presence of just a finite number of defects. This task is simpler, although in practice one has to limit the treatment to the level of one or at most two lowest orders in $n_{i}$. An important assumption made in the development of this approach is that all integrations in Eq. (A4), in every term of expansion in $n_{i}$, do not lead to divergences. Convergence must be maintained for all parameters in the set $\{u\}$ and all values of $\alpha(T)$ especially the point $\alpha(T)=0$. This property, ensuring that this method of expansion is controlled, is far from being a forgone conclusion, and must be carefully addressed once the specific form of the defect potential is chosen. As will be shown below, for the essentially onedimensional potentials such as those given by Eq. (11), the convergence is maintained for all $\alpha(T)$ in the first order of expansion in $n_{i}$. Although, we will be concerned below only with this lowest order, we believe that the procedure is well-behaved at all orders, as long as defects are parallel to each other and the one-dimensional character of the problem is maintained. 


\section{SUPERFLUID DENSITY}

In this Section, we calculate the superfluid density in the presence of randomly located planar defects based on the expansion in powers of defect concentration $n_{i}$. We will limit ourselves to calculations up to first order in $n_{i}$. It is useful, however, to first discuss the qualitative behavior of the superconducting order parameter in the presence of defects, without specifying the dimensionality of the problem or the form of the potential $u\left(\mathbf{r}-\mathbf{R}_{i}\right)$. In the absence of randomness, the superfluid density is 15

$$
\rho_{s}^{(0)}(T)=\left[\Psi_{0}^{(0)}(T)\right]^{2}=\left\{\begin{array}{cc}
0, & \alpha(T)>0 \\
|\alpha(T)| / b, & \alpha(T)<0
\end{array}\right.
$$

To calculate the first order correction in $n_{i}$ to this result, one needs to solve the saddle point equation for the order parameter $\Psi_{0}^{(1)}\left(\mathbf{R}_{i} ; \mathbf{r}\right)$ in the presence of one defect located at point $\mathbf{R}_{i}$ :

$$
\left[-\frac{\nabla^{2}}{2 m}+\alpha(T)+u\left(\mathbf{r}-\mathbf{R}_{i}\right)\right] \Psi_{0}^{(1)}\left(\mathbf{R}_{i} ; \mathbf{r}\right)+b\left|\Psi_{0}^{(1)}\left(\mathbf{R}_{i} ; \mathbf{r}\right)\right|^{2} \Psi_{0}^{(1)}\left(\mathbf{R}_{i} ; \mathbf{r}\right)=0 .
$$

It is clear that $\Psi_{0}^{(1)}\left(\mathbf{R}_{i} ; \mathbf{r}\right)=\Psi_{0}^{(1)}\left(\mathbf{r}-\mathbf{R}_{i}\right)$, and without loss of generality we can consider the defect to be located at $\mathbf{R}_{i}=0$. The non-zero real and positive solution of this equation occurs at the point where the eigenvalue spectrum of the operator $\hat{L}_{0}[u]$, containing a single-defect potential, reaches zero. In analogy with Eq. (6),

$$
\hat{L}_{0}[u]=-\frac{\nabla^{2}}{2 m}+\alpha(T)+u(\mathbf{r})
$$

Provided that the solution obtained from Eq. (14) is stable, we can write down the general formula for the superfluid density averaged over the randomness in $u(\mathbf{r})$

$$
\overline{\rho_{s}}(T)=\left\{\begin{array}{cc}
n_{i} \int_{\mathcal{C}} \tilde{\mathcal{P}}(\{u\}) \mathcal{D}\{u\} \int\left[\Psi_{0}^{(1)}(\mathbf{r})\right]^{2} d \mathbf{r}, & \alpha(T)>0 \\
|\alpha(T)| / b+n_{i} \int_{\mathcal{C}} \tilde{\mathcal{P}}(\{u\}) \mathcal{D}\{u\} \int\left\{\left[\Psi_{0}^{(1)}(\mathbf{r})\right]^{2}-|\alpha(T)| / b\right\} d \mathbf{r}, & \alpha(T)<0 .
\end{array}\right.
$$

Expression Eq. (16) follows straightforwardly from Eq. (12), applied to the square of the order parameter. But the same result can be obtained using time-dependent Ginzburg-Landau equation as a starting point 15 One needs to calculate the transverse response, and subsequently perform the average using the same method of expansion in powers of $n_{i}$ in the long wavelength limit ${ }^{17}$ The meaning of notations $\int_{\mathcal{C}}$ and $\tilde{\mathcal{P}}(\{u\})$ in Eq. (16) will be discussed below. We only mention now that we must carefully integrate, not over all possible values of parameters from the set $\{u\}$, but only over those realizations that, first, give stable solutions for $\Psi_{0}^{(1)}(\mathbf{r})$ and, second, result in a defect-affected critical temperature not exceeding some fixed value $T_{c}$.

If the system contains defects that give rise to a stable positive non-zero solution of Eq. (14) at $T$ greater than $T_{c}^{(0)}$, superconductivity must be presumed shifted to higher temperatures. In this case, a finite transverse response will be observed above the critical temperature of the homogeneous sample. To first order in $n_{i}$, the actual transition point will be determined by the defect that leads to the strongest enhancement of $T_{c}$ in the sample. This means that in experiments, the temperature $T_{c}$, at which the superconducting response is first seen will be disorder-dependent and non-universal. Very close to $T_{c}$, the superfluid density will be tiny, since the order parameter will be determined by contributions coming from a very small number of defects. But with decreasing temperature, the fraction of defects giving rise to non-zero solutions of Eq. (14) will increase, leading to an increase in the superconducting response. At $T>T_{c}^{(0)}$, the solutions for the order parameter, $\Psi_{0}^{(1)}\left(\mathbf{r}-\mathbf{R}_{i}\right)$, will be localized around the center of the defect at point $\mathbf{R}_{i}$. Indeed, as long as $\alpha \equiv \alpha(T)>0$, in the absence of any defects, the only stable solution for the order parameter is zero. Hence, it follows from Eq. (14) that for functions $u(\mathbf{r})$ that vanish quickly enough with distance,

$$
\Psi_{0}^{(1)}(\mathbf{r})=\frac{g_{>}(\mathbf{r})}{\sqrt{b}} e^{-\sqrt{2 m \alpha} r}
$$

when the condition $\sqrt{m \alpha} r \gg 1$ is satisfied. The function $g_{>}(\mathbf{r})$ which has a weaker than exponential dependence on $r$, is determined by the effective dimensionality of the problem. For instance, in two dimensions $g_{>}(\mathbf{r})=1 / \sqrt{r}$ and does not contain any variables related to the potential. 18 For $\sqrt{m \alpha} r \leq 1$, however, the functional form of the decay of the order parameter is no longer exponential and depends strongly on the non- 
universal characteristics of the function $u(\mathbf{r})$. Eq. (17) also describes the behavior of $\Psi_{0}^{(1)}(\mathbf{r})$ at all distances when $\alpha=0$ exactly. The corresponding asymptotic forms are in fact written out in Table 1 of Ref. 18 and we will not discuss them further here.

When $T<T_{c}^{(0)}$ and $\alpha<0$, the stable solution for the clean system is given by $\Psi_{0}^{(0)}(T)=\sqrt{|\alpha| / b}$, and we expect from Eq. (14), that finite values of $u(\mathbf{r})$ will add some perturbation to this solution that falls off at infinity. We look for solutions of the form

$$
\Psi_{0}^{(1)}(\mathbf{r})=\frac{1}{\sqrt{b}}[\sqrt{|\alpha|}+\psi(\mathbf{r})]
$$

where the real auxiliary function $\psi(\mathbf{r})$ satisfies the equation

$$
\begin{array}{r}
{\left[-\frac{\nabla^{2}}{2 m}+2|\alpha|+u(\mathbf{r})\right] \psi(\mathbf{r})+\sqrt{|\alpha|} u(\mathbf{r})} \\
+3 \sqrt{|\alpha|}[\psi(\mathbf{r})]^{2}+[\psi(\mathbf{r})]^{3}=0 .
\end{array}
$$

By analogy to Eq. (17) and provided that $\sqrt{m|\alpha|} r \gg 1$, we can write

$$
\psi^{(1)}(\mathbf{r})=g_{<}(\mathbf{r}) e^{-2 \sqrt{m|\alpha|} r}
$$

with the function $g_{<}(\mathbf{r})$ having an asymptotic form similar to that of $g_{>}(\mathbf{r})$. Again, closer to the defect when $\sqrt{m|\alpha|} r \sim 1$, the crossover to a different functional form, with stronger dependence on characteristics of the potential, will take place. Note also that the requirement for $\Psi_{0}^{(1)}(\mathbf{r})$ to be positive, does not prevent the function $g_{<}(\mathbf{r})$ from having both signs. This means that depending on the form and sign of $u(\mathbf{r})$, the order parameter may be either enhanced or suppressed in the vicinity of a defect for $\alpha<0$. Thus we conclude that the spatial variation of the order parameter changes qualitatively when $\alpha$ passes through zero, meaning that the defects play a different role in the system above and below $T_{c}^{(0)}$. Above $T_{c}^{(0)}$, only a portion of all defects will perturb the zero value of $\Psi_{0}^{(0)}$ and, though widely scattered, they nevertheless ensure a small but finite superconducting response. At the same time, below $T_{c}^{(0)}$, every defect will affect the solution $\Psi_{0}^{(0)}=\sqrt{|\alpha| / b}$, but this just leads to a correction to the superfluid density that becomes more and more innocuous with decreasing temperature. In some sense, we can say that, because of these qualitative differences, the point $T=T_{c}^{(0)}$ acts as a special kind of critical point. Indeed, a simple inspection of Eq. (16) reveals that, despite the continuity of the superfluid density at $\alpha=0$, its derivative with respect to temperature exhibits a jump.
It is then appropriate to ask what kind of a defect potential $u\left(\mathbf{r}-\mathbf{R}_{i}\right)$ leads to an increase in the critical temperature. To answer this question, consider the eigenvalue problem for the operator $\hat{L}_{0}[u]-\alpha$, where $\hat{L}_{0}[u]$ is given by Eq. (15):

$$
\left[-\frac{\nabla^{2}}{2 m}+u\left(\mathbf{r}-\mathbf{R}_{i}\right)\right] \chi_{\epsilon}\left(\mathbf{r}-\mathbf{R}_{i}\right)=\epsilon \chi_{\epsilon}\left(\mathbf{r}-\mathbf{R}_{i}\right) .
$$

This equation is nothing other than the Schrödinger equation determining the stationary states of a particle moving in the presence of potential $u\left(\mathbf{r}-\mathbf{R}_{i}\right)$. For a potential which falls off rapidly enough at infinity, the spectrum of positive eigenvalues $\epsilon$ is continuous. It is describable by a number of quantum variables, with $k=\sqrt{2 m \epsilon}$ being one of them. The spectrum of negative eigenvalues $E_{n}$, if any exist, is discrete. All eigenvalues $\epsilon$ are explicit functions of all parameters in the set $\{u\}$. It is easy to see then that the transition point, in the presence of one defect, is determined by the smallest eigenvalue, $E_{0}$, of the operator $\hat{L}_{0}[u]-\alpha$. From the condition $\alpha-\left|E_{0}\right|=0$ and Eq. (3) it follows that, because of the defect, $T_{c}=T_{c}^{(0)}\left(1+\left|E_{0}\right| / a\right)$. If there are no discrete levels, $E_{0}=0$ and no increase of the critical temperature occurs. We note that the points of instability coincide with the poles of the Green's function of the operator $\hat{L}_{0}[u]$, obeying the following equation

$$
\hat{L}_{0}[u] \mathcal{G}^{(1)}\left(\mathbf{R}_{i} ; \mathbf{r}, \mathbf{r}^{\prime}\right)=\delta\left(\mathbf{r}-\mathbf{r}^{\prime}\right)
$$

The superscript means that the Green's function are calculated in the presence of only one defect located at $\mathbf{R}_{i}$. Above $T_{c}$,

$$
\mathcal{G}^{(1)}\left(\mathbf{R}_{i} ; \mathbf{r}, \mathbf{r}^{\prime}\right)=\sum_{\{\epsilon\}} \frac{\chi_{\epsilon}\left(\mathbf{r}-\mathbf{R}_{i}\right) \chi_{\epsilon}^{\star}\left(\mathbf{r}^{\prime}-\mathbf{R}_{i}\right)}{\alpha+\epsilon}
$$

where $\sum_{\{\epsilon\}}$ formally denotes the summation and integration over the discrete and continuous branches of the spectrum. Since any defect breaks translational invariance, $\mathcal{G}^{(1)}\left(\mathbf{R}_{i} ; \mathbf{r}, \mathbf{r}^{\prime}\right)$ depends separately on $\mathbf{r}$ and $\mathbf{r}^{\prime}$ rather than on $\mathbf{r}-\mathbf{r}^{\prime}$. The Green's function in the momentum representation $\mathcal{G}^{(1)}\left(\mathbf{R}_{i} ; \mathbf{p}, \mathbf{p}^{\prime}\right)$ is the Fourier transform of Eq. (23). It contains two momenta, $\mathbf{p}$ and $\mathbf{p}^{\prime}$, and has the same pole structure but may be more straightforward to calculate depending on the form of potential.

Following the same strategy that led to Eq. (12), it is possible to write down the Green's function averaged over the positions of defects and parameters of their potentials. In analogy with Eq. (12), up to first order in $n_{i}$ : 


$$
\overline{\mathcal{G}^{(N)}\left(\left\{u_{1}\right\} \mathbf{R}_{1} \ldots\left\{u_{N}\right\} \mathbf{R}_{N} ; \mathbf{r}, \mathbf{r}^{\prime}\right)}=\mathcal{G}^{(0)}\left(\mathbf{r}-\mathbf{r}^{\prime}\right)+n_{i} \int \mathcal{P}(\{u\}) \mathcal{D}\{u\} \int\left[\mathcal{G}^{(1)}\left(\{u\} ; \mathbf{R} ; \mathbf{r}, \mathbf{r}^{\prime}\right)-\mathcal{G}^{(0)}\left(\mathbf{r}-\mathbf{r}^{\prime}\right)\right] d \mathbf{R}
$$

An approximation that contains only the first power in $n_{i}$ is known as a single-site approximation $19,20,21$. If all defects have one and the same potential, the system is considered to have binary disorder, in which case the integration over $\mathcal{D}\{u\}$ would be absent in Eqs.(12), (24). For this widely studied simplified type of randomness, the Green's function Eq. (24) is obtainable as a result of resummation of a certain class of diagrams $\frac{19,22}{}$. If the distribution of parameters characterizing the potentials $u\left(\mathbf{r}-\mathbf{R}_{i}\right)$ is broad enough, averaging over them with the weight $\mathcal{P}(\{u\})$ plays an important role introducing an additional complicating ingredient to the problem. The critical value of $\alpha(T)$ for which the non-zero solution of Eq. (14) first appears in this case, will properly coincide with the singularity in the disorder averaged Green's function as can be seen from Eqs. (23), (24). It will be determined by the defect that induces the maximum local $T_{c}$ in the sample.

Next we address the question of the stability of possible solutions of Eq. (14). In the presence of one defect (presumed located at $\mathbf{R}_{i}=0$ ), the solution is stable if the eigenspectrum of operators $\hat{L}_{\perp}[u]$ and $\hat{L}_{\|}[u], \varepsilon_{\perp}$ and $\varepsilon_{\|}$, written in analogy with Eqs. (7) and (8), is non-negative. That is, one needs to analyze two equations:

$$
\begin{aligned}
& {\left[-\frac{\nabla^{2}}{2 m}+\alpha+u(\mathbf{r})+b\left[\Psi_{0}^{(1)}(\mathbf{r})\right]^{2}\right] f_{\perp}(\mathbf{r})=\varepsilon_{\perp} f_{\perp}(\mathbf{r}),} \\
& {\left[-\frac{\nabla^{2}}{2 m}+\alpha+u(\mathbf{r})+3 b\left[\Psi_{0}^{(1)}(\mathbf{r})\right]^{2}\right] f_{\|}(\mathbf{r})=\varepsilon_{\|} f_{\|}(\mathbf{r}) .}
\end{aligned}
$$

To do this, we employ the following general mathematical result for the spectra of second-order differential operators. The eigenvalues can be ordered in a sequence of increasing values, and the eigenfunction corresponding to the lowest eigenvalue (the ground state eigenfunction) has no nodes as a function of $\mathbf{r} \stackrel{23}{s}$ Eigenfunctions corresponding to higher energies must change sign somewhere in space and are orthogonal to the ground state eigenfunction. Comparing Eqs. (14) and (25), we see that the eigenfunction corresponding to $\varepsilon_{\perp}=0$ is given by $\Psi_{0}^{(1)}(\mathbf{r})$. Hence if it is everywhere positive, we can claim that it is the ground state of operator $\hat{L}_{\perp}[u]$. This result immediately implies the conclusion that the lowest eigenvalue of Eq. (26), $\varepsilon_{\| 0}$, cannot be negative. Indeed, the ground state eigenfunction $f_{\| 0}(\mathbf{r})$ must be bounded and can not change sign anywhere. Hence if we consider the ground states of Eqs. (25) and (26), multiply them respectively by $f_{\| 0}(\mathbf{r})$ and $\Psi_{0}^{(1)}(\mathbf{r})$, integrate over $d \mathbf{r}$ and then subtract the first from the second, we find that

$$
2 b \int\left[\Psi_{0}^{(1)}(\mathbf{r})\right]^{3} f_{\| 0}(\mathbf{r}) d \mathbf{r}=\varepsilon_{\| 0} \int \Psi_{0}^{(1)}(\mathbf{r}) f_{\| 0}(\mathbf{r}) d \mathbf{r} .
$$

This immediately implies that $\varepsilon_{\| 0}>0$ is the only possibility, and hence that all other $\varepsilon_{\|}>0$ as well. This result is just a simple manifestation of the fact that, for predominantly positive potentials, the set of eigenvalues shifts up. We can not say, however, whether the ground state belongs to the discrete spectrum or lies at the bottom edge of the continuous one. From Eq. (26) it follows that for $\alpha>0$, the continuous spectrum starts at $\varepsilon_{\|}=\alpha$, while for $\alpha<0, \varepsilon_{\|}=2|\alpha|$ is its lowest possible eigenvalue. If $0<\varepsilon_{\| 0}<\alpha$ and $\alpha>0$ (or $2|\alpha|$ for $\alpha<0)$, then the ground state eigenfunction $f_{\| 0}(\mathbf{r})$ belongs to the discrete branch of the spectrum and falls off exponentially at infinity. But if $\varepsilon_{\| 0}$ is the lowest possible eigenvalue of the continuous spectrum, $f_{\| 0}(\mathbf{r})$ tends to some non-zero constant as $|\mathbf{r}| \rightarrow \infty$. Similar analysis of asymptotics following from Eq. (25) leads to the result that for $\alpha<0$, the spectrum of $\hat{L}_{\perp}[u]$ is purely continuous and starts right from the ground state zeroth eigenvalue. If $\alpha>0$, however, one can not exclude the presence of some additional energy levels belonging to the discrete spectrum in the segment $0<\varepsilon_{\perp}<\alpha$. To conclude, if one finds a solution of Eq. (14) $\Psi_{0}^{(1)}(\mathbf{r})$ positive for all $\mathbf{r}$, it is guaranteed to be stable. We are not aware of any general analytic methods that allow us to solve Eq. (14) because of the cubic non-linearity. But the possibility that solutions may be found, depending on the relation between $\alpha$ and the parameters of $u(\mathbf{r})$, seems quite realistic for smooth potentials of a given sign which decay monotonically at infinity.

We are now in the position to discuss how to perform the average over disorder realizations $\int_{\mathcal{C}}$ in Eq. (16), and what limitations one should impose on the distribution of randomness in order to obtain physically sensible results for the superfluid density. As discussed earlier, our approach implies that the actual critical temperature is determined by the defect which gives the greatest increase of $T_{c}^{(0)}$. This approximation is a consequence of considering the problem in the lowest order in $n_{i}$ and suggests that, to compare the theoretically calculated disordersmeared behavior with experimental data, the actual $T_{c}$ should be introduced by hand. This can be achieved if the integral $\int \mathcal{D}\{u\}$ is performed, not over all possible values from the set $\{u\}$, but rather over those of them that do not allow for the defect-shifted critical temperatures greater than the stipulated $T_{c}$. Subscript $\mathcal{C}$ in Eq. (16) is used to indicate exactly that. Since in this case $\int_{\mathcal{C}} \mathcal{P}(\{u\}) \mathcal{D}\{u\}<1$, it is appropriate to introduce the normalized distribution,

$$
\tilde{\mathcal{P}}(\{u\})=\frac{1}{\mathcal{A}} \mathcal{P}(\{u\}), \quad \mathcal{A}=\int_{\mathcal{C}} \mathcal{P}(\{u\}) \mathcal{D}\{u\}
$$


and employ it in the average over disorder potentials.

In addition, to eliminate the possibility of unphysical behavior of the superfluid density as a function of temperature, the disorder distribution must be regarded as symmetric about its average. Namely, we must require the fulfillment of the constraint:

$$
\int_{\mathcal{C}} \mathcal{P}(\{u\}) \mathcal{D}\{u\} \int u(\mathbf{r}) d \mathbf{r}=0 .
$$

To clarify its importance, we first note that the positive sign of $\overline{\rho_{s}}$ does not follow automatically from Eq. (14) when $\alpha<0$. Indeed, if we divide Eq. (14) by $b \Psi_{0}^{(1)}(\mathbf{r}-$ $\mathbf{R}_{i}$ ) and integrate over space and $\mathcal{D}\{u\}$, then using Eq. (16) for $\alpha<0$, we find that

$$
\begin{gathered}
\overline{\rho_{s}}=\frac{|\alpha|}{b}+ \\
\frac{n_{i}}{b} \int_{\mathcal{C}} \mathcal{P}(\{u\}) \mathcal{D}\{u\} \int\left[\frac{\nabla^{2} \Psi_{0}^{(1)}(\mathbf{r})}{2 m \Psi_{0}^{(1)}(\mathbf{r})}-u(\mathbf{r})\right] d \mathbf{r}
\end{gathered}
$$

We can then integrate by parts the term containing $\Psi_{0}^{(1)}(\mathbf{r})$, with the help of Eqs. (18), (20). Hence, if Eq. (28) is satisfied, it follows that

$$
\overline{\rho_{s}}=\frac{|\alpha|}{b}+\frac{n_{i}}{2 m b} \int_{\mathcal{C}} \mathcal{P}(\{u\}) \mathcal{D}\{u\} \int\left[\frac{\nabla \Psi_{0}^{(1)}(\mathbf{r})}{\Psi_{0}^{(1)}(\mathbf{r})}\right]^{2} d \mathbf{r}(30)
$$

The integrand in Eq. (30) is always positive, ensuring that $\overline{\rho_{s}}>0$ everywhere below $T_{c}^{(0)}$. This could not be the case, had the left hand side of Eq. (28) been negative. Although nothing wrong occurs if it is positive, it is convenient to ensure that Eq. (28) is satisfied by adjusting $T_{c}^{(0)}$ which so far has been assumed to be the transition temperature of a disorder-free sample.

We now apply this general formalism to the case in which the defects are described by the potential:

$$
u(\mathbf{r})=-u \delta\left(x-X_{i}\right)
$$

As mentioned in the Introduction, our model consists of a stack of parallel planes that locally change the critical temperature. The planes are infinite in $y$ and $z$ directions, resulting in an essentially a one-dimensional problem. Eq. (31) also implies that the planes are formally of zero thickness. Physically, this corresponds to a situation in which the actual width of the planar defects is of order $\xi_{0}$. The only new parameter in the problem having dimensions of length is $1 /(m|u|)$ which, together with the correlation length $\xi=1 / \sqrt{2 m \alpha}$, determines the character of the solution for the order parameter. Comparing those two length scales one can see that any shift to $\alpha$ should be proportional to $m u^{2}$. The order parameter $\Psi_{0}^{(1)}(\mathbf{r})$ depends only on $x$, and assuming, again without loss of generality, that the defect is at the origin, we write the equation

$$
\left[-\frac{1}{2 m} \frac{d^{2}}{d x^{2}}+\alpha-u \delta(x)\right] \Psi_{0}^{(1)}(x)+b\left[\Psi_{0}^{(1)}(x)\right]^{3}=0 .
$$

The corresponding solution $\Psi_{0}^{(1)}(x)$ must be continuous, but its first derivative has a jump at $x=0$, meaning that

$$
\left.\frac{d \Psi_{0}^{(1)}(x)}{d x}\right|_{x=+0}-\left.\frac{d \Psi_{0}^{(1)}(x)}{d x}\right|_{x=-0}=-2 m u \Psi_{0}^{(1)}(0)
$$

As has been discussed above, two qualitatively different solutions are possible depending on the sign of $\alpha$, and we must consider separately two cases.

\section{A. Case $\alpha>0$}

In this case, the solution and its first derivative must decrease exponentially at infinity. Elementary integration then leads to the result that 22,24

$$
\Psi_{0}^{(1)}(x)=\frac{\sqrt{2 \alpha}}{\sqrt{b} \sinh \left[\sqrt{2 m \alpha}|x|+\lambda_{1}\right]},
$$

where the constant $\lambda_{1}$, determined from the condition of Eq. (33), is given by

$$
\lambda_{1}=\operatorname{arctanh} \frac{1}{u} \sqrt{\frac{2 \alpha}{m}}
$$

Note that the solution Eq. (34) makes sense only if $u>0$. At large $|x|$,

$$
\Psi_{0}^{(1)}(x) \approx \sqrt{\frac{8 \alpha}{b}}\left(\frac{\sqrt{m u^{2}}-\sqrt{2 \alpha}}{\sqrt{m u^{2}}+\sqrt{2 \alpha}}\right)^{1 / 2} \cdot e^{-\sqrt{2 m \alpha}|x|},
$$




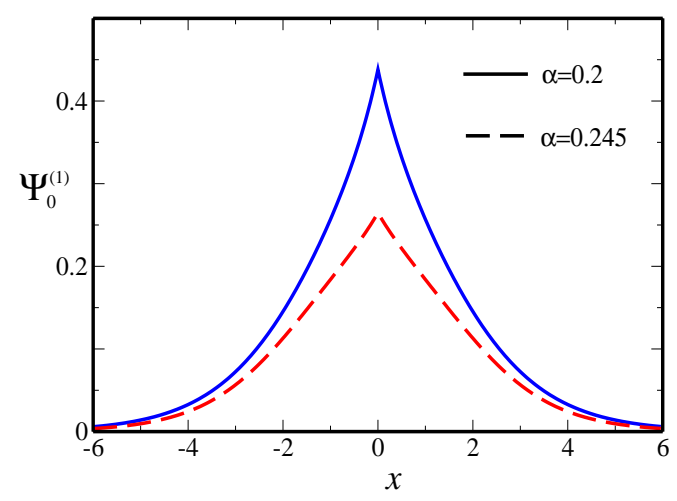

FIG. 1: (Color online). Order parameter $\Psi_{0}^{(1)}$ plotted as a function of $x$ using Eq. (34) for $m=2.0, b=1.0$ and $u=0.5$ and two values of $\alpha$ shown in the figure. in agreement with Eq. (17), while at $x=0$ :

$$
\Psi_{0}^{(1)}(0)=\sqrt{\frac{m u^{2}-2 \alpha}{b}}
$$

This solution is possible only if $0<2 \alpha<m u^{2}$. If $2 \alpha>m u^{2}$, the only stable solution is $\Psi_{0}^{(1)}(x)=0$. We conclude that the amount by which $u$ increases the critical temperature is connected to $\alpha$ by

$$
\alpha=\frac{m u^{2}}{2}
$$

It is instructive to check this result by calculating explicitly the Green's function form Eq. (22) and finding its poles. It is convenient to work in the momentum representation. Since the problem is translationally invariant in the $y$ and $z$ directions, we seek solutions of the form

$$
\mathcal{G}^{(1)}\left(X_{i}=0 ; \mathbf{r}, \mathbf{r}^{\prime}\right)=\frac{1}{(2 \pi)^{2}} \int_{-\infty}^{\infty} \int_{-\infty}^{\infty} e^{i\left(q_{y} y+q_{z} z\right)} d q_{y} d q_{z} \int_{-\infty}^{\infty} \int_{-\infty}^{\infty} e^{i\left(p x+p^{\prime} x^{\prime}\right)} \mathcal{G}^{(1)}\left(\eta_{y z} ; p, p^{\prime}\right) d p d p^{\prime}
$$

where $\eta_{y z} \equiv \eta\left(q_{y}, q_{z}\right)=q_{y}^{2} /(2 m)+q_{z}^{2} /(2 m)$. Substituting into Eq. (22) and performing simple manipulations in the term involving the $\delta$-function, we obtain two equations to be solved self-consistently

$$
\mathcal{G}^{(1)}\left(\eta_{y z} ; p, p^{\prime}\right)=\frac{\delta\left(p+p^{\prime}\right)+u C_{1}\left(p^{\prime}\right)}{\alpha+\eta_{y z}+p^{2} /(2 m)}, \quad C\left(p^{\prime}\right)=\int_{-\infty}^{\infty} \mathcal{G}^{(1)}\left(\eta_{y z} ;-p^{\prime \prime}, p^{\prime}\right) \frac{d p^{\prime \prime}}{2 \pi}
$$

The result for the full Green's function is

$$
\mathcal{G}^{(1)}\left(\eta_{y z} ; p, p^{\prime}\right)=\frac{\delta\left(p+p^{\prime}\right)}{\alpha+\eta_{y z}+p^{2} /(2 m)}+\frac{u}{2 \pi[1-u K]} \cdot \frac{1}{\left[\alpha+\eta_{y z}+p^{2} /(2 m)\right]\left[\alpha+\eta_{y z}+\left(p^{\prime}\right)^{2} /(2 m)\right]},
$$

in terms of the integral

$$
\begin{aligned}
K \equiv K\left(\eta_{y z}\right) & =\int_{-\infty}^{\infty} \frac{d p^{\prime \prime}}{2 \pi} \cdot \frac{1}{\alpha+\eta_{y z}+\left(p^{\prime \prime}\right)^{2} /(2 m)} \\
& =\frac{m}{\sqrt{2 m\left(\alpha+\eta_{y z}\right)}} .
\end{aligned}
$$

The first term in Eq. (41) is the Green's function corresponding to the absence of any potential, and has the simple pole at $\alpha=0$. The second term is the nontranslationally invariant contribution due to the presence of the defect. For positive $u$ only, the factor containing $K$ has an additional pole at $\alpha=m u^{2} / 2$, implying an increase of the critical temperature in agreement with Eq. (38).
B. Case $\alpha<0$

In this case, as $|x| \rightarrow \infty, \Psi_{0}^{(1)}(x) \rightarrow \sqrt{|\alpha| / b}$. It is easy to complete the integration to obtain 22.24

$$
\begin{gathered}
\Psi_{0}^{(1)}(x)=\left\{\begin{array}{c}
\sqrt{|\alpha| / b} \operatorname{coth}\left[\sqrt{m|\alpha|}|x|+\lambda_{2}\right], u>0, \\
\sqrt{|\alpha| / b} \tanh \left[\sqrt{m|\alpha|}|x|+\lambda_{2}\right], u<0,
\end{array}\right. \\
\lambda_{2}=\frac{1}{2} \operatorname{arcsinh} \frac{1}{|u|} \sqrt{\frac{4|\alpha|}{m}} .
\end{gathered}
$$

For $\alpha<0$, potentials with both signs of $u$ lead to physically sensible positive solutions. At large distances, $\sqrt{m|\alpha|}|x| \gg 1$,

$$
\Psi_{0}^{(1)}(x) \approx \sqrt{\frac{|\alpha|}{b}}\left(1+\frac{m u \cdot e^{-2 \sqrt{m|\alpha|}|x|}}{\sqrt{|\alpha|}+\sqrt{|\alpha|+m u^{2} / 4}}\right),
$$



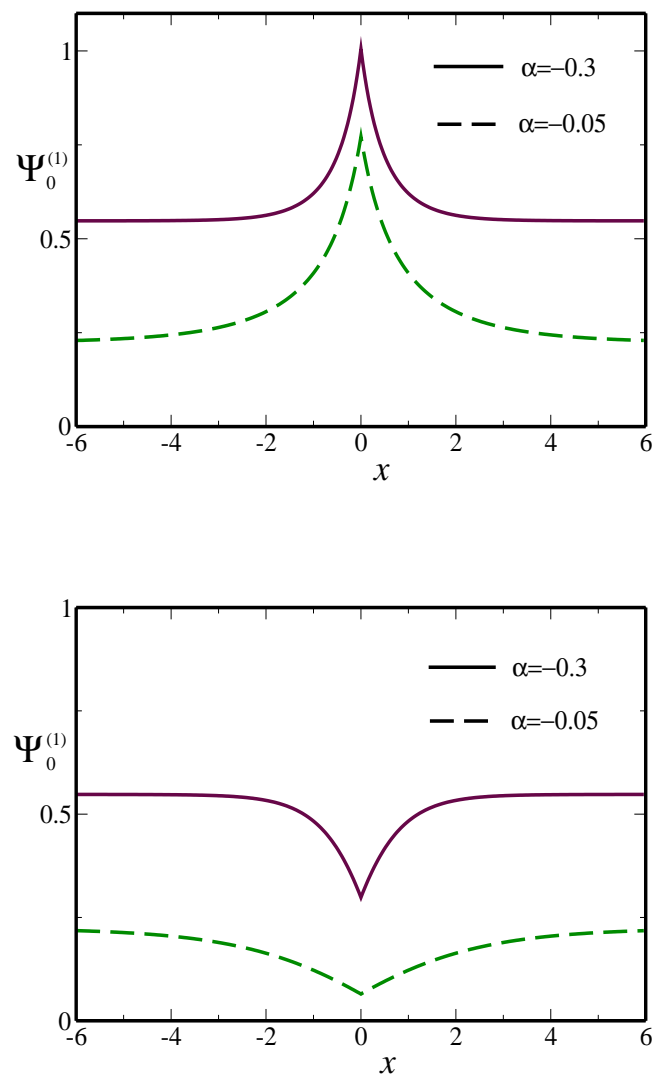

FIG. 2: (Color online). Order parameter $\Psi_{0}^{(1)}$ plotted as a function of $x$ using Eq. (43) for $m=2.0, b=1.0$ and $u=0.5$ (upper figure), $u=-0.5$ (lower figure). The values of $\alpha$ corresponding to each curve are displayed in the figures.

which has the asymptotic form discussed in the previous Section. Full expressions for $\Psi_{0}^{(1)}(0)$ can be derived in a straight forward manner, but here we only present the less cumbersome ones in the limit of large and small (with respect to $\left.m u^{2}\right)|\alpha|$,

$$
\Psi_{0}^{(1)}(0) \approx \sqrt{\frac{|\alpha|}{b}}\left(1+\frac{u}{2} \sqrt{\frac{m}{|\alpha|}}\right), \quad m u^{2} \ll|\alpha| ;
$$

$$
\Psi_{0}^{(1)}(0) \approx\left\{\begin{aligned}
\sqrt{\frac{m}{b}} \cdot \frac{|\alpha|}{m|u|}, & u<0, \\
\sqrt{\frac{m}{b}} u, & u>0,
\end{aligned} \quad m u^{2} \gg|\alpha|\right.
$$

Depending on the sign of $u$, the order parameter is either greater or smaller than $\Psi_{0}^{(0)}(x)=\sqrt{|\alpha| / b}$. Thus, if $\alpha$ tends to zero from below, the solution for the order parameter vanishes if $u<0$, but transforms into

$$
\Psi_{0}^{(1)}(x)=\sqrt{\frac{m}{b}} \frac{u}{m u|x|+1}, \quad \alpha=0,
$$

for positive $u$. The dependence on $x$ becomes a power law, meaning that the influence of the defect is long range at the special point $\alpha=0$.

We can now substitute the solutions given by Eqs. (34), (43) into Eq. (16) and calculate the average superfluid density $\overline{\rho_{s}}(T) . u$ is taken to obey the symmetric Gaussian distribution,

$$
P[u]=\frac{1}{\sqrt{2 \pi} W} \exp \left\{-\frac{u^{2}}{2 W^{2}}\right\}
$$

with mean $W$. This form of distribution implies an exponentially rare probability of occurrence for defects with potentials with strength much greater than average. However, since $u$ can, in principle, take any value in the distribution Eq. (49), the defect-induced enhancement of the critical temperature is formally unbounded. We must then impose an upper limit $u_{m}$ on possible values of $u$, which will define the actual critical temperature

$$
T_{c}=T_{c}^{(0)}\left(1+\frac{m u_{m}^{2}}{2 a}\right) .
$$

The renormalized distribution to be used in Eq. (16) is

$$
\tilde{\mathcal{P}}(\{u\})=P[u] / \mathcal{A}, \quad \mathcal{A}=\int_{-u_{m}}^{u_{m}} P[u] d u
$$

and it is simple to integrate over $x$ and obtain

$$
\overline{\rho_{s}}(T)=\left\{\begin{array}{cl}
\frac{2 n_{i}}{b} \int_{\sqrt{2 \alpha / m}}^{u_{m}} \frac{P[u]}{\mathcal{A}}\left(u-\sqrt{\frac{2 \alpha}{m}}\right) d u, & \alpha>0, \\
\frac{|\alpha|}{b}+\frac{2 n_{i}}{b} \sqrt{\frac{|\alpha|}{m}} \int_{0}^{u_{m}} \frac{P[u]}{\mathcal{A}} \cdot \frac{\left(\operatorname{coth} \lambda_{2}-1\right)^{2}}{\operatorname{coth} \lambda_{2}} d u, & \alpha<0 .
\end{array}\right.
$$

In deriving the result for $\alpha<0$, we explicitly used the

symmetry, $P[-u]=P[u]$. Noting that, from Eq. (44),

$$
\operatorname{coth} \lambda_{2}=\left(\frac{\sqrt{4|\alpha|+m u^{2}}+\sqrt{m}|u|}{\sqrt{4|\alpha|+m u^{2}}-\sqrt{m}|u|}\right)^{1 / 2},
$$


we see that for $\alpha=0$ the two expressions in Eq. (52) become identical. The presence of the $T_{c}^{(0)}$-enhancing defects makes the superfluid density finite at that point. Estimating its order of magnitude, we can write

$$
\overline{\rho_{s}}\left(T_{c}^{(0)}\right) \sim \frac{n_{i} W}{b} .
$$

The value in the righthand side of Eq. (54) contains the first power of $n_{i}$ and is presumed small enough so that the whole approach based on the Ginzburg-Landau expansion remains valid.

For convenience, we introduce the rescaled parameters

$$
\sqrt{\frac{m}{2}} u \rightarrow u, \quad \sqrt{\frac{m}{2}} W \rightarrow W, \quad \sqrt{\frac{2}{m}} n_{i} \rightarrow n_{i}
$$

and plot $b \overline{\rho_{s}}(T)$ as a function of $\alpha(T)=a\left(T-T_{c}^{(0)}\right) / T_{c}^{(0)}$, assuming $a=1$. The results for several values of disorder distribution width $W(W=0.0, W=0.1$, and $W=0.2$ ) and $u_{m}=3 W$ on all plots, are presented in Fig. 3 .

From the plots it follows that, if the values of $u$ are broadly distributed and $u_{m} \gg W$ belongs to the Lifshitz tail, the superfluid density for larger $\alpha$ is exponentially small. Under the same circumstances, the behavior near the point $T=T_{c}^{(0)}$ is not sensitive to the exact value of $u_{m}$. We note also that for $u_{m} \gg W, \mathcal{A} \approx 1$ and is not of much importance. Below $T_{c}^{(0)}$, however, the behavior asymptotically approaches that of the disorderfree system. These results are in qualitative agreement with the solution for the order parameter presented in Ref. 22, having a small but finite value going to zero at some weakly $n_{i}$-dependent value $T_{c}>T_{c}^{(0)}$.

In Fig. 4. we present data for experimental measurements of the superfluid density taken from Ref. 14. The upper figure shows the results for the superfluid density of an $\mathrm{YBa}_{2} \mathrm{Cu}_{3} \mathrm{O}_{6.333}$ ellipsoid, measured as a function of temperature for different values of doping starting from the most ordered sample with $T_{c}=17 K$. The lower figure describes the behavior of $\rho_{s}(T)$ very close to the critical temperature for two levels of doping that lead to the highest $T_{c}$ 's $(\approx 17 K$ and $\approx 15 K)$ as shown in the upper figure. In experiments described in Ref. 14, the superfluid density was determined from the penetration depth measurements done on the high quality crystals. Highly ordered samples were prepared by extended annealing under pure oxygen gas flow, as well as high hydrostatic pressure at room temperature to enhance $\mathrm{CuO}$-chain ordering ${ }^{25,26}$. This procedure removes randomness in the spatial distribution of isolated oxygen vacancies that could act as pinning and scattering centers. However, planar-type inhomogeneities are present as twin boundaries as these materials are not detwinned. In conventional superconductors, the twin boundaries are well known to increase locally the critical temperature 13 . In $\mathrm{YBa}_{2} \mathrm{Cu}_{3} \mathrm{O}_{6.333}$, the twin boundaries parallel to the $c$-axis are envisioned as the planes separating two regions in which the $\mathrm{CuO}$ chains are oriented perpendicular to each other, and are likely to serve as a source of
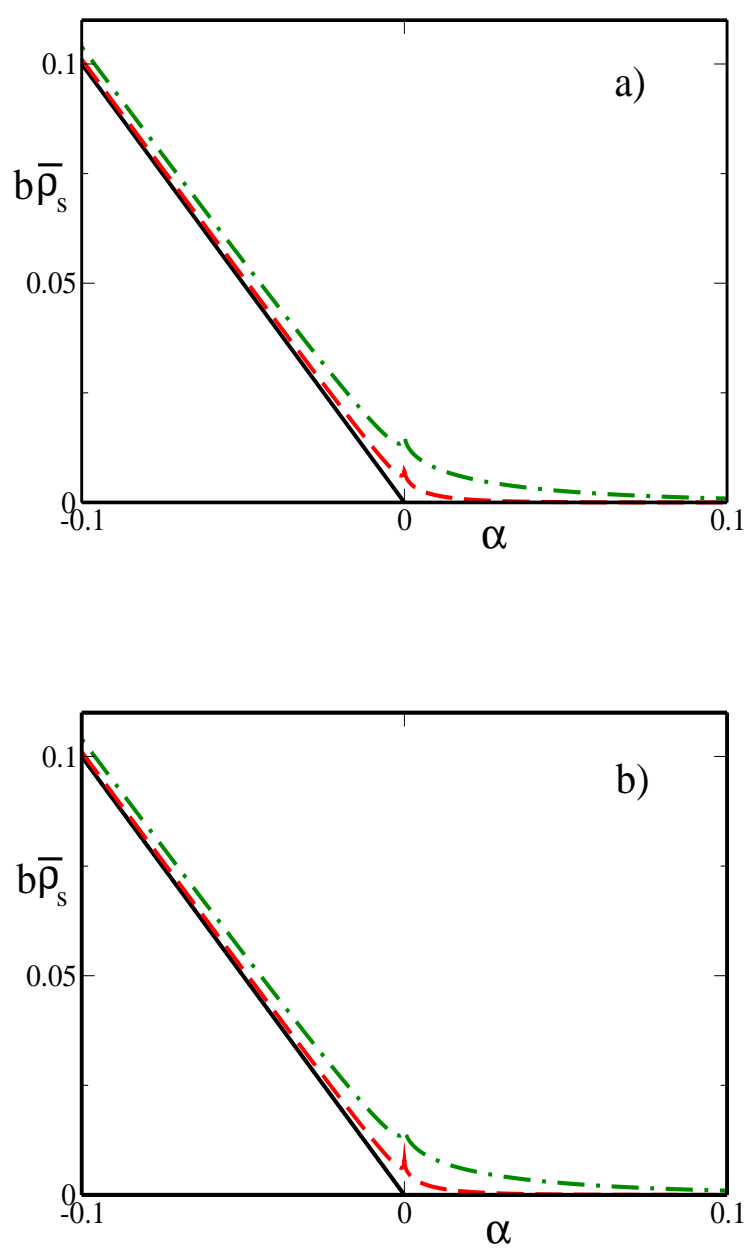

FIG. 3: (Color online). The figures show the dependence of $b \overline{\rho_{s}}(T)$ as a function of $\alpha(T)=a\left(T-T_{c}^{(0)}\right) / T_{c}^{(0)}(a=1)$ calculated from Eq. (52) for the rescaled by means of Eq. (55) parameters $n_{i}=0.1, u_{m}=3 W$ for figure a) and $n_{i}=0.1$, $u_{m}=5 \mathrm{~W}$ for figure b) respectively. The widths of the disorder distribution $W=0.0, W=0.1, W=0.2$ in both figures, are represented by the solid, dashed and dashed-dotted lines respectively.

$T_{c}$-enhancement as well. The reason for this is that it is advantageous for the oxygen vacancies to be located near the twin boundaries 27 . And the presence of narrow regions with surplus of these vacancies means the enhancement of superconductivity, and as a consequence higher critical temperature in the vicinity. For each plane, this is effectively modeled by adding the potential Eq. (31) to the conventional GL action. Randomness of $u$ is likely to come from variation of the in-plane concentration of vacancies, stemming in its turn from the non-uniformity of the initial density of the twin boundaries. Since, in our case all such planar defects appear to increase the critical temperature, the values of $u$ are determined relative 

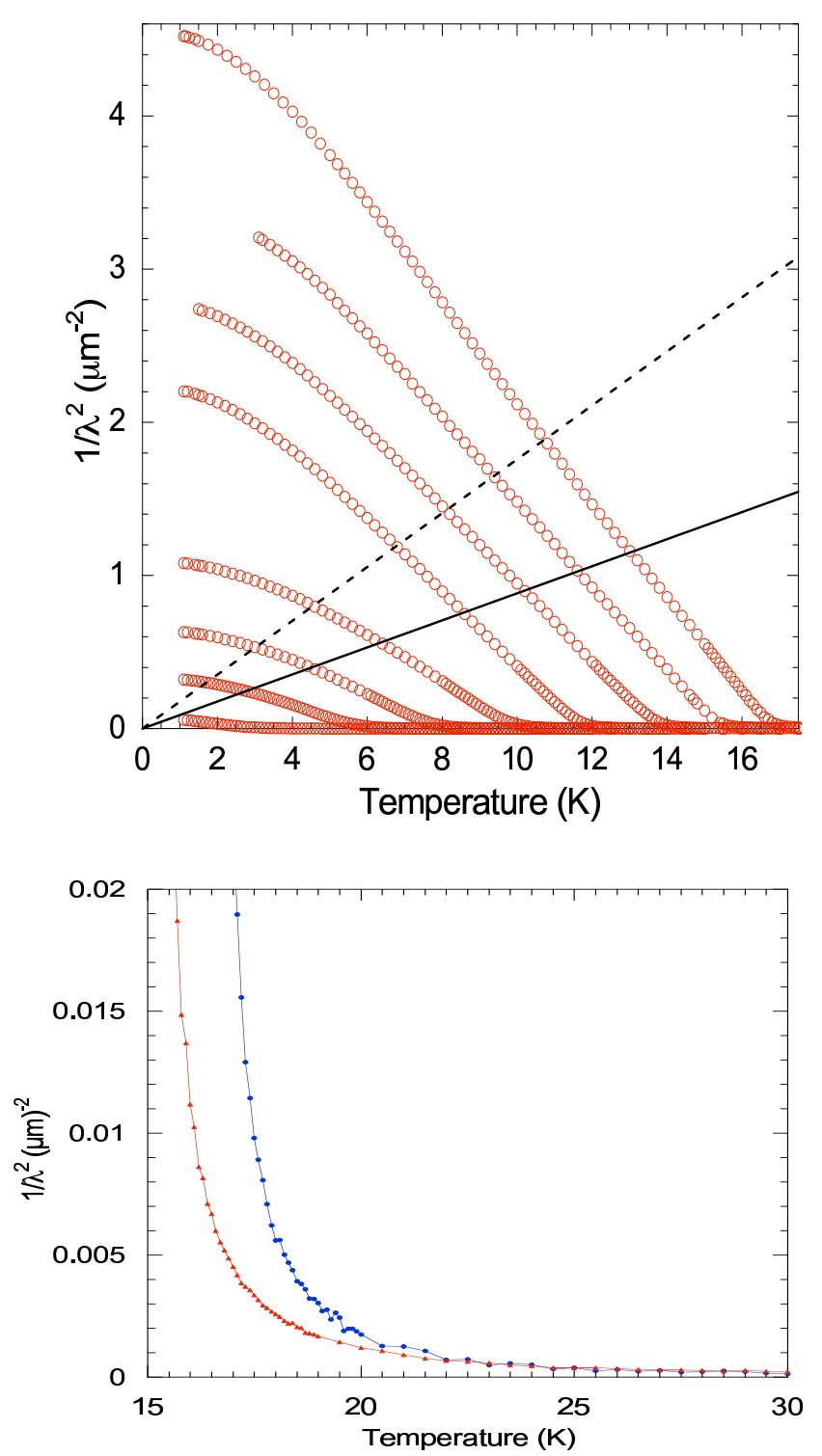

FIG. 4: Measured superfluid density in the underdoped regime of $\mathrm{YBa}_{2} \mathrm{Cu}_{3} \mathrm{O}_{6.333}$ taken from Ref. 14. The data suggest the presence of decreasing superconducting response above some temperature. The meaning of the curves is explained in the text.

to some average value, hence having both signs. $T_{c}^{(0)}$ defined previously as the critical temperature of completely disorder-free sample must thus be regarded shifted up so that Eq. (28) is satisfied. We should mention also that the issue of local superconductivity enhancement due to the twin-boundaries themselves has been considered previously in Ref. 28. The twinning planes were assumed to form a periodic array and be described in the GL functional by the sum of terms of the form Eq. (1) all having the same $u$. In our approach, we specifically highlight the importance of randomness in potentials for the purpose of the qualitative interpretation of the measured superfluid density.
It is seen from the data in Fig. 4 that a small but finite superfluidity persists above some temperature obtained by extrapolating the straight lines, describing the behavior at lower $T$ down to zero. If we associate this value with $T_{c}^{(0)}$ in our approach, we can claim that the simple model of planar disorder reproduces qualitatively the experimentally observed temperature dependence of $\rho_{s}$. From the data, though, it is difficult to infer the precise value of the actual critical temperature $T_{c}$. Since the aim of this calculation is mainly to illustrate the generic features resulting from randomness in $T_{c}$ 's, we have not attempted to determine the choices of parameters $W, u_{m}, a$ required for a precise match between the theoretical and experimental curves. Already this qualitative agreement suggests that even in experiments done on high quality samples, there are local regions with critical temperatures significantly above the average $T_{c}$. The smallness of superfluid density in the tails suggests that such regions are rare and have broadly distributed local critical temperatures.

\section{DISCUSSION AND CONCLUSIONS}

In this Section, we discuss the relevance of the simple model of random planar defects to the experimentally observed behavior of the superfluid density as a function of temperature close to $T_{c}$.

First, it is appropriate to ask what changes to the behavior of $\rho_{s}$ are expected if we go to the next orders of expansion in defect concentration. To examine this, consider how one proceeds to obtain the correction to the superfluid density that is of second order in $n_{i}$. According to the general strategy, it is necessary to solve the equation for the order parameter in the presence of two defects located at points $X_{1}$ and $X_{2}$. The distance $L=\left|X_{2}-X_{1}\right|$ emerges as a new parameter for the problem, and together with the strengths of the potentials will determine the point at which the real positive solution $\Psi_{0}^{(2)}(L ; x)$ first obtains. To find it, one can easily solve the corresponding generalization of Eq. (22) for the Green's function $\mathcal{G}^{(2)}\left(X_{1}=0, X_{2}=L ; \mathbf{r}, \mathbf{r}^{\prime}\right)$ of the operator $\hat{L}_{0}[u]$ with the potential

$$
u(\mathbf{r})=-u_{1} \delta(x)-u_{2} \delta(x-L)
$$

The subscript (2) means now that we do all calculations in the presence of exactly two defects. The solution has the form

$$
\begin{gathered}
\mathcal{G}^{(2)}\left(\eta_{y z} ; p, p^{\prime}\right)= \\
\frac{\delta\left(p+p^{\prime}\right)+u_{1} C_{1}\left(p^{\prime}\right)+u_{2} C_{2}\left(p^{\prime}\right) e^{-i p L}}{\alpha+\eta_{y z}+p^{2} /(2 m)} \\
C_{1}\left(p^{\prime}\right)=\int_{-\infty}^{\infty} \mathcal{G}^{(2)}\left(\eta_{y z} ;-p^{\prime \prime}, p^{\prime}\right) \frac{d p^{\prime \prime}}{2 \pi}
\end{gathered}
$$




$$
C_{2}\left(p^{\prime}\right)=\int_{-\infty}^{\infty} e^{-i p^{\prime \prime} L} \mathcal{G}^{(2)}\left(\eta_{y z} ;-p^{\prime \prime}, p^{\prime}\right) \frac{d p^{\prime \prime}}{2 \pi} .
$$

We will not write out in full the cumbersome expression for the Green's function and state only that the poles of $\mathcal{G}^{(2)}\left(\eta_{y z} ; p, p^{\prime}\right)$, indicating the occurrence of a transition at some $T>T_{c}^{(0)}$, are determined from the equation

$$
\begin{array}{r}
\left(1-u_{1} \sqrt{\frac{m}{2 \alpha}}\right)\left(1-u_{2} \sqrt{\frac{m}{2 \alpha}}\right)- \\
\frac{m u_{1} u_{2}}{2 \alpha} \cdot e^{-2 \sqrt{2 m \alpha}|L|}=0 .
\end{array}
$$

The left hand side of Eq. (59) is just the corresponding determinant (taken at $\eta_{y z}=0$ ) which arises in the process of solving the system of two coupled linear equations for $C_{1}\left(p^{\prime}\right)$ and $C_{2}\left(p^{\prime}\right)$. If $u_{1}=u_{2}$, Eq. (59) reduces (upon the proper rescaling) to the known result 24 . It is clear from Eq. (59), that for positive $u_{1}$ and $u_{2}$, the critical value of $\alpha=(m / 2) \max \left(u_{1}^{2}, u_{2}^{2}\right)$ if $|L|=\infty$, implying a complete independence of the defects. But for $|L|=0$, the pole occurs at $\alpha=(m / 2)\left(u_{1}+u_{2}\right)^{2}$, in agreement with the fact that if both defects are located at one point, their strengths simply add. It follows then that placing the second defect with positive $u$ at any finite distance $L$, in addition to the one already present, increases the critical $\alpha$. This means that if there are two defects located not too far from each other in the sample, with strengths close to $u_{m}$, the actual critical temperature, as a result of going to the second order in $n_{i}$, will be higher than that given by Eq. (50). However, this circumstance does not affect the qualitative interpretation of the data since, as was mentioned before, the presumed exponential smallness of $\rho_{s}(T)$ at temperatures considerably higher than $T_{c}^{(0)}$, renders the actual $T_{c}$ difficult to determine from Fig. 4. Thus we will not discuss further all the calculations to second and higher orders in $n_{i}$, but rather note the following. Once the potential strengths are broadly distributed, the value of $b \overline{\rho_{s}}(T)$ at $T=T_{c}^{(0)}$ is not sensitive to the actual $T_{c}$. To calculate it one can safely set $u_{m}=\infty$. However, the calculated $b \overline{\rho_{s}}(T)$ using Eq. (52) has a peak at $\alpha=0$ as seen in Fig. 3. We believe that this non-monotonic behavior in the vicinity of $T_{c}^{(0)}$, is an artifact of the mean-field approximation used in our treatment from the very beginning. Thermal fluctuations when $\alpha$ is small, are expected to renormalize down the values of the superfluid density, but consideration of this question is beyond the scope of this paper.

The planar defects considered in this paper, may not be the only ones present despite the high quality of the samples. Isolated and rare point defects due to oxygen disorder, other lattice defects such as dislocations 29 are, in principle, not excluded ${ }^{30}$. It is important, however, that among all possible types of defects, the plane-like ones ensure the broadest possible distribution of the local critical temperatures. This follows from the generalized Harris criterion ${ }^{1,31}$, arguing that whenever $2-d^{*} \nu>0$, with $\nu$ being the correlation length critical exponent and $d^{*}$ the number of dimensions in which the system is random, disorder is relevant near the critical point. For point and columnar disorder, $d^{*}$ is equal to 3 and 2 respectively, whereas in the case of stacked planar defects $d^{*}=1$. Obviously, the greater $2-d^{*} \nu$ is, the wider the distribution of local $T_{c}$ 's is due to randomness in parameters characterizing the defect potential ${ }^{32}$. One should remember, though, that not all defects, but only the extended ones of sizes greater than $\xi_{0}$, can be satisfactorily accounted for within the framework of GL theory. Others should be treated using a suitable microscopic model. If necessary, possibly in lower quality samples, defects with spherical and cylindrical shapes must be considered as well, but these are likely to affect the superfluid density in a much narrower region around $T_{c}^{(0)}$. Investigation of such defects is left for future work.

\section{Acknowledgments}

The authors would like to thank Prof. David Broun for many helpful conversations and for providing the data presented in the figures above. CK and AJB are supported by the Natural Sciences and Engineering Research Council of Canada, by the Canadian Foundation for Innovation and by the Canadian Institute for Advanced Research.

\section{APPENDIX A: EXPANSION IN POWERS OF $n_{i}$}

In this Appendix, we obtain the average of the function $F_{N}\left(\left\{u_{1}\right\} \mathbf{R}_{1}, \ldots,\left\{u_{N}\right\} \mathbf{R}_{N} ; \mathbf{r}\right)$ given by Eq. (10) in the form of a series in powers of defect concentration $n_{i}$. The corresponding derivation is straightforward and follows the lines of Ref. 16. We should notice first, however, that the function in angular brackets in Eq. (10) is not symmetric, but the final result will not change if we replace $F_{N}\left(\left\{u_{1}\right\} \mathbf{R}_{1}, \ldots,\left\{u_{N}\right\} \mathbf{R}_{N} ; \mathbf{r}\right)$ with the function

$$
F_{s N}\left(\left\{u_{1}\right\}, \ldots,\left\{u_{N}\right\} ; \mathbf{R}_{1}, \ldots, \mathbf{R}_{N} ; \mathbf{r}\right)=\frac{1}{N !} \hat{P}\left[\left\{u_{1}\right\}, \ldots,\left\{u_{N}\right\}\right] F_{N}\left(\left\{u_{1}\right\} \mathbf{R}_{1}, \ldots,\left\{u_{N}\right\} \mathbf{R}_{N} ; \mathbf{r}\right)
$$


symmetrized over all sets $\left\{u_{i}\right\}$ for a particular location of the defect.( $\hat{P}$ is the symmetrization operator) This creates functions symmetric with respect to permutations of coordinates $\mathbf{R}_{1}, \ldots, \mathbf{R}_{N}$. It is possible to verify then that for any finite number of such functions $F_{s m}\left(\left\{u_{1}\right\}, \ldots,\left\{u_{m}\right\} ; \mathbf{R}_{1}, \ldots, \mathbf{R}_{m} ; \mathbf{r}\right),(m \geq 0)$

$$
\begin{aligned}
& F_{s N}\left(\left\{u_{1}\right\}, \ldots,\left\{u_{N}\right\} ; \mathbf{R}_{1}, \ldots, \mathbf{R}_{N} ; \mathbf{r}\right)=F_{0}(\mathbf{r})+\sum_{i} \Phi_{1}\left(\left\{u_{i}\right\} ; \mathbf{R}_{i} ; \mathbf{r}\right) \\
& \quad+\sum_{i<j}\left[\Phi_{s 2}\left(\left\{u_{i}\right\},\left\{u_{j}\right\} ; \mathbf{R}_{i}, \mathbf{R}_{j} ; \mathbf{r}\right)-\Phi_{1}\left(\left\{u_{i}\right\} ; \mathbf{R}_{i} ; \mathbf{r}\right)-\Phi_{1}\left(\left\{u_{j}\right\} ; \mathbf{R}_{j} ; \mathbf{r}\right)\right] \\
& +\sum_{i<j<k}\left[\Phi_{s 3}\left(\left\{u_{i}\right\},\left\{u_{j}\right\},\left\{u_{k}\right\} ; \mathbf{R}_{i}, \mathbf{R}_{j}, \mathbf{R}_{k} ; \mathbf{r}\right)-\Phi_{s 2}\left(\left\{u_{i}\right\},\left\{u_{j}\right\} ; \mathbf{R}_{i}, \mathbf{R}_{j} ; \mathbf{r}\right)-\Phi_{s 2}\left(\left\{u_{i}\right\},\left\{u_{k}\right\} ; \mathbf{R}_{i}, \mathbf{R}_{k} ; \mathbf{r}\right)\right. \\
& \left.\quad-\Phi_{s 2}\left(\left\{u_{j}\right\},\left\{u_{k}\right\} ; \mathbf{R}_{j}, \mathbf{R}_{k} ; \mathbf{r}\right)+\Phi_{1}\left(\left\{u_{i}\right\} ; \mathbf{R}_{i} ; \mathbf{r}\right)+\Phi_{1}\left(\left\{u_{j}\right\} ; \mathbf{R}_{j} ; \mathbf{r}\right)+\Phi_{1}\left(\left\{u_{k}\right\} ; \mathbf{R}_{k} ; \mathbf{r}\right)\right]+\cdots
\end{aligned}
$$

In Eq. (A2) $(m \geq 2)$,

$$
\Phi_{s m}\left(\left\{u_{1}\right\}, \ldots,\left\{u_{m}\right\} ; \mathbf{R}_{1}, \ldots, \mathbf{R}_{m} ; \mathbf{r}\right)=F_{s m}\left(\left\{u_{1}\right\}, \ldots,\left\{u_{m}\right\} ; \mathbf{R}_{1}, \ldots, \mathbf{R}_{m} ; \mathbf{r}\right)-F_{0}(\mathbf{r})
$$

and these $\Phi_{s m}\left(\left\{u_{1}\right\}, \ldots,\left\{u_{m}\right\} ; \mathbf{R}_{1}, \ldots, \mathbf{R}_{m} ; \mathbf{r}\right)$ are also symmetric. $F_{0}(\mathbf{r})$ is a function of $\mathbf{r}$ only, and it, as well as $\Phi_{1}\left(\left\{u_{i}\right\} ; \mathbf{R}_{i} ; \mathbf{r}\right)$, does not require symmetrization. Assuming that all defects are located at points $\mathbf{R}_{i}$, and that $F_{0}(\mathbf{r})$ is the value in the absence of defects, we can take the thermodynamic limit $N \rightarrow \infty, V \rightarrow \infty, n_{i}=N / V$ in Eq. (A2) to get

$$
\begin{gathered}
\left\langle F_{N}\left(\left\{u_{1}\right\} \mathbf{R}_{1}, \ldots,\left\{u_{N}\right\} \mathbf{R}_{N} ; \mathbf{r}\right)\right\rangle=F_{0}(\mathbf{r})+n_{i} \int \Phi_{1}\left(\left\{u_{1}\right\} ; \mathbf{R}_{1} ; \mathbf{r}\right) d \mathbf{R}_{1} \\
+\frac{n_{i}^{2}}{2 !} \iint\left[\Phi_{s 2}\left(\left\{u_{1}\right\},\left\{u_{2}\right\} ; \mathbf{R}_{1}, \mathbf{R}_{2} ; \mathbf{r}\right)-\Phi_{1}\left(\left\{u_{1}\right\} ; \mathbf{R}_{1} ; \mathbf{r}\right)-\Phi_{1}\left(\left\{u_{2}\right\} ; \mathbf{R}_{2} ; \mathbf{r}\right)\right] w_{2}\left(\left\{u_{1}\right\},\left\{u_{2}\right\} ; \mathbf{R}_{2}-\mathbf{R}_{1}\right) d \mathbf{R}_{1} d \mathbf{R}_{2} \\
+\frac{n_{i}^{3}}{3 !} \iiint\left[\Phi_{s 3}\left(\left\{u_{1}\right\},\left\{u_{2}\right\},\left\{u_{3}\right\} ; \mathbf{R}_{1}, \mathbf{R}_{2}, \mathbf{R}_{3} ; \mathbf{r}\right)-\Phi_{s 2}\left(\left\{u_{1}\right\},\left\{u_{2}\right\} ; \mathbf{R}_{1}, \mathbf{R}_{2} ; \mathbf{r}\right)-\Phi_{s 2}\left(\left\{u_{2}\right\},\left\{u_{3}\right\} ; \mathbf{R}_{2}, \mathbf{R}_{3} ; \mathbf{r}\right)\right. \\
\left.-\Phi_{s 2}\left(\left\{u_{3}\right\},\left\{u_{1}\right\} ; \mathbf{R}_{3}, \mathbf{R}_{1} ; \mathbf{r}\right)+\Phi_{1}\left(\left\{u_{1}\right\} ; \mathbf{R}_{1} ; \mathbf{r}\right)+\Phi_{1}\left(\left\{u_{2}\right\} ; \mathbf{R}_{2} ; \mathbf{r}\right)+\Phi_{1}\left(\left\{u_{3}\right\} ; \mathbf{R}_{3} ; \mathbf{r}\right)\right] \\
w_{3}\left(\left\{u_{1}\right\},\left\{u_{2}\right\},\left\{u_{3}\right\} ; \mathbf{R}_{2}-\mathbf{R}_{1}, \mathbf{R}_{3}-\mathbf{R}_{2}\right) d \mathbf{R}_{1} d \mathbf{R}_{2} d \mathbf{R}_{3}+\cdots
\end{gathered}
$$

The full average then follows in a straightforward way by substituting this average over positions into Eq. (10). In Eq. (A4), $w_{2}\left(\left\{u_{1}\right\},\left\{u_{2}\right\} ; \mathbf{R}_{2}-\mathbf{R}_{1}\right)$ is the probability of the simultaneous occurrence of two defects at points $\mathbf{R}_{1}$ and $\mathbf{R}_{2}$ characterized by the sets $\left\{u_{1}\right\}$ and $\left\{u_{2}\right\}$ respectively. Obviously, $w_{2}\left(\left\{u_{1}\right\},\left\{u_{2}\right\} ; \mathbf{R}_{2}-\mathbf{R}_{1}\right)=w_{2}\left(\left\{u_{1}\right\},\left\{u_{2}\right\} ; \mathbf{R}_{1}-\mathbf{R}_{2}\right)$. Probabilities $w_{3}, w_{4}, \ldots$ in higher order terms have analogous meaning, and in general depend on $\left\{u_{i}\right\}$. However, this dependence is likely to be noticeable only if defects are close to each other. All functions $w_{m}$ will depend on concentration $n_{i}$, but in the limit all $\left|\mathbf{R}_{i}-\mathbf{R}_{k}\right| \gg 1 /\left(n_{i}\right)^{1 / d}$, they quickly tend to unity. Those functions must also factorize if one of the coordinates tends to infinity, meaning, for example, that $w_{3}\left(\left\{u_{1}\right\},\left\{u_{2}\right\},\left\{u_{3}\right\} ; \mathbf{R}_{2}-\mathbf{R}_{1}, \mathbf{R}_{3}-\right.$ $\left.\mathbf{R}_{2}\right) \rightarrow w_{2}\left(\left\{u_{1}\right\},\left\{u_{3}\right\} ; \mathbf{R}_{3}-\mathbf{R}_{1}\right)$, if $\left|\mathbf{R}_{2}\right| \rightarrow \infty$.

1 A. B. Harris,J. Phys. C 7, 1671, 1974.

2 I M Lifshitz, Sov. Phys.-JETP 17 1159, 1963.

${ }^{3}$ M Franz et al. Phys. Rev. B 56, 7882-7885 (1997).

4 A. A. Abrikosov and L. P. Gorkov, Sov. Phys. JETP 12, 1243 (1961).

${ }^{5}$ R. J. Radtke et al. Phys. Rev. B 48, 653 (1993).

6 A. I. Larkin and Yu. N. Ovchinnikov, Sov. Phys. JETP 34, 651 (1972).

7 L. B. Ioffe and A. I. Larkin, Sov. Phys. JETP 54, 378 (1982).

8 K. Machida, T. Koyama, and T. Matsubara, Phys. Rev. B 23, 99 (1981).

9 C. A. Balseiro and L. M. Falicov, Phys. Rev. B 20, 4457 (1979).
10 M. Vojta and S. Sachdev, Phys. Rev. Lett. 83, 3916 (1999).

11 S. A. Kivelson and V. J. Emery, in Strongly Correlated Electronic Materials: The Los Alamos Symposium 1993 ed. by K. S. Bedell, Z.Wang, B. E. Meltzer, A.V. Balatsky, and E. Abrahams (Addison-Wesley, Redding, 1994).

12 E. W. Carlson, V. J. Emery, S. A. Kivelson, and D. Orgad, in The Physics of Conventional and Unconventional Superconductors, edited by K. H. Bennemann and J. B. Ketterson (Springer-Verlag, Berlin, 2002).

13 I. N. Khlyustikov and A. I. Buzdin, Adv. in Phys. 36, 271 (1987).

14 D.M. Broun, P.J. Turner, W.A. Huttema, S. Ozcan, B. Morgan, R. Liang, W.N. Hardy and D.A. Bonn, Phys. Rev. Lett. 99, 237003 (2007). 
15 H.Schmidt, Z. Physik 232, 443 (1970); Z. Physik, 216, 336 (1970).

16 I.M. Lifshitz, S. A. Gredeskul and L. A. Pastur, Introduction to the Theory of Disordered Systems, Wiley, New York, 1968; Ch.5.

17 D. Dalidovich, (unpublished), and there is strong evidence that the higher local critical temperature is tied to the increased concentration of these vacancies.

18 A. J. Millis, D. K. Morr, and J. Schmalian, Phys. Rev. Lett. 87, 167202 (2001).

19 R.J. Elliott, J.A. Krumhansl, and P.L. Leath, Rev. Mod. Phys. 46, 465 (1974).

20 J. S. Langer, Phys. Rev. 120, 714 (1960).

21 J.S. Langer, J. Math. Phys. 2, 584 (1961).

${ }^{22}$ F. Schwabl and U.C. Tauber, Phys. Rev. B 43, 11112 (1991).

23 E. C. Titchmarsh, Eigenfunction expansions Associated with Second-order Differential Equations (Oxford University Press, London, 1958)
${ }^{24}$ H. Schmidt and F. Schwabl, Z. Phys. B 30, 197 (1978).

25 Ruixing Liang, W. N. Hardy, and D. A. Bonn, Physica C 336, 57 (2000).

26 R. Liang, D. A. Bonn, W. N. Hardy, J. C. Wynn, K. A. Moler, L. Lu, S. Larochelle, L. Zhou, M. Greven, L. Lurio and S. G. J. Mochrie, Physica C 383, 1 (2002).

27 Adam C. Durst and Patrick A. Lee, Phys. Rev. B 65, 094501 (2002).

28 A. A. Abrikosov, A. I. Buzdin, M. L. Kulic and D. A. Kuptsov, Zh. Eksp. Teor. Fiz. 95, 371 (1989) [Sov. Phys. JETP 68, 210 (1989)].

29 V. M. Nabutovskii and V. Ya. Shapiro, Zh. Eksp. Teor. Fiz. 75, 948 (1978) [Sov. Phys. JETP 48, 480 (1978)].

30 A. J. Berlinsky, D. A. Bonn, R. Harris, and C. Kallin, Phys. Rev. B 61, 9088 (2000).

31 M. Wallin, E. S. Sørensen, S. M. Girvin, and A. P. Young, Phys. Rev. B 49, 12115 (1994)

32 T. Vojta, J. Phys. A 36, 10291 (2003). 